\title{
La recherche de nouveaux matériaux thermoélectriques
}

Sylvie Hébert (sylvie.hebert@ensicaen.fr)

Laboratoire CRISMAT, UMR 6508 CNRS et ENSICAEN, 6 Bd du Maréchal Juin, 14050 Caen Cedex

Les effets thermoélectriques permettent de transformer une différence de température $\Delta \mathrm{T}$ en une différence de potentiel électrique $\Delta \mathrm{V}$ via l'effet Seebeck, ou $\Delta \mathrm{V}$ en $\Delta \mathrm{T}$ via l'effet Peltier. Ces deux effets sont de bonnes sondes de la structure électronique des matériaux, et peuvent également être utilisés pour transformer la chaleur perdue en électricité (mode Seebeck) ou pour fabriquer des refroidisseurs (mode Peltier).

Limitée pour le moment à des secteurs très spécialisés comme le spatial, la thermoélectricité connaît un regain d'intérêt depuis les années 1990 - 2000, suite à différentes propositions visant à améliorer les performances des matériaux thermoélectriques.

Remerciements

Je souhaite remercier mes collègues du laboratoire CRISMAT qui contribuent à l'étude de ces matériaux thermoélectriques (oxydes, sulfures et séléniures), ainsi que Jiri Hejtmanek (Institute of Physics, Prague), Véronique Brouet, Julien Bobroff et Philippe Mendels (LPS Orsay), et Patrice Limelette (GREMAN Tours) pour leur collaboration sur ce sujet.
Les effets thermoélectriques ont été découverts au cours du $19^{\mathrm{e}}$ siècle. Les deux plus " célèbres » sont l'effet Seebeck et l'effet Peltier (encadré 1), mais il existe de nombreux autres effets thermoélectriques, associés à l'application simultanée d'un champ magnétique ou d'un courant électrique, tels l'effet Nernst, l'effet Thomson ou l'effet Ettinghausen [1]. Ces différents effets thermoélectriques sont très étudiés en physique de la matière condensée, car ce sont des propriétés de transport extrêmement sensibles à la structure électronique du matériau (encadré 2). Ils constituent de bonnes sondes d'étude des porteurs, de leur densité d'états et de leurs différents mécanismes de diffusion (diffusion des charges sur le réseau atomique via les phonons, diffusion par les spins, diffusion entre charges...).

La conversion $\Delta \mathrm{T} \rightarrow \Delta \mathrm{V}$ via l'effet Seebeck ou $\Delta \mathrm{V} \rightarrow \Delta \mathrm{T}$ via l'effet Peltier permet de plus d'envisager des applications de ces matériaux dans les domaines de la production d'électricité ou de la réfrigération. L'efficacité d'un module thermoélectrique pour ces applications est rappelée dans l'encadré 3 , où est défini le "facteur de mérite » ZT. Elle est comparée au rendement de Carnot (rendement idéal d'un dispositif thermodynamique fonctionnant entre une température chaude $T_{c}$ et une température froide $T_{f}$ et défini comme $\left.\left(T_{c}-T_{f}\right) / T_{c}\right)$. Pour des modules Seebeck, le rendement maximal peut atteindre $10 \%$ du rendement de Carnot pour $\Delta \mathrm{T}=700 \mathrm{~K}$, avec $\mathrm{T}_{\mathrm{f}}=300 \mathrm{~K}$ et $\mathrm{ZT}=1(18 \%$ si $\mathrm{ZT}=2)$.

À l'heure actuelle, les modules thermoélectriques sont employés dans des domaines de niche, tels que l'espace ou les zones éloignées des circuits classiques de distribution électrique (en montagne, pour des systèmes de communication sur des pipelines...). Ces générateurs sont très utilisés dans les applications spatiales, en particulier ceux à base de $\mathrm{PbTe}$ ou de SiGe embarqués dès 1961 par la NASA, ou plus récemment pour la mission Curiosity vers Mars. Dans ce domaine, la technologie thermoélectrique a montré toute son efficacité, puisque des thermogénérateurs fonctionnent depuis 30 ans, avec de faibles pertes de puissance, par exemple dans les sondes Voyager. De plus, un intérêt de cette technique est que les modules ne sont soumis à aucun mouvement mécanique et n'utilisent pas de fluide (contrairement à la réfrigération classique), rendant cette technologie très fiable et robuste. Une autre application à grande échelle est la climatisation de sièges de voitures aux États-Unis et au Canada par refroidisseurs Peltier : seuls les sièges utilisés sont actifs, ce qui évite de climatiser tout l'habitacle du véhicule.

Toute chaleur perdue est aussi potentiellement une source d'électricité grâce à des thermogénérateurs Seebeck, dont l'utilisation parait très attractive comme nouvelle source d'énergie "propre ". La thermoélectricité a connu un regain d'intérêt depuis une quinzaine d'années, et de nombreux projets sont en cours d'étude. Ainsi, il est possible d'équiper des cuisinières, des chauffe-eaux ou des lampes de thermogénérateurs, servant ensuite à alimenter de façon autonome de petits équipements électriques. Le corps humain et les gradients thermiques à sa surface peuvent également servir à alimenter des capteurs de surveillance médicale. En Europe et aux États-Unis, différents projets existent dans le domaine automobile pour recycler la chaleur en sortie du pot d'échappement. Dans le domaine de la microélectronique, la dissipation thermique des composants est le paramètre essentiel qui limite l'amélioration des performances, et des composants à effet Peltier pourraient être utilisés dans la gestion de la chaleur perdue.

L'efficacité d'un matériau (et donc d'un module) dépend de son facteur de mérite ZT. Les trois grandeurs définissant ZT 


\section{Effet Seebeck et effet Peltier}

encadré 1

Lorsque les deux bornes d'un matériau sont soumises à une différence de température $\Delta \mathrm{T}$, une différence de potentiel électrique $\Delta \mathrm{V} y$ apparaît : c'est l'effet Seebeck, découvert en 1823 par Thomas Johann Seebeck. Le coefficient Seebeck est défini à une température $T$ par $\mathrm{S}=\Delta \mathrm{V} / \Delta \mathrm{T}$, dans la limite $\Delta \mathrm{T} \rightarrow 0$. Inversement, lorsqu'un matériau est soumis à une différence de potentiel $\Delta \mathrm{V}$, une différence de température $\Delta \mathrm{T}$ apparaît à ses bornes, via l'effet Peltier, découvert en 1834 par Jean-Charles Athanase Peltier. Ces deux effets peuvent être utilisés pour fabriquer des thermogénérateurs qui vont générer une tension électrique à partir de la chaleur perdue (mode Seebeck, fig. E1a), ou inversement refroidir des matériaux ou des composants électroniques (mode Peltier, fig. E1b).

Les effets thermoélectriques sont décrits par la thermodynamique des processus irréversibles. Une description très simplifiée est que lorsqu'un matériau est soumis à une différence de température $\Delta T$, les porteurs de charge du côté chaud se déplacent vers le côté froid pour revenir vers un état d'équilibre, créant un courant de diffusion. Pour un matériau en circuit ouvert, ce courant est nul ; il y a donc création $\mathrm{d}^{\prime}$ une tension $\Delta \mathrm{V}$, s'opposant à l'établissement de ce déplacement, tension à l'origine du coefficient Seebeck.

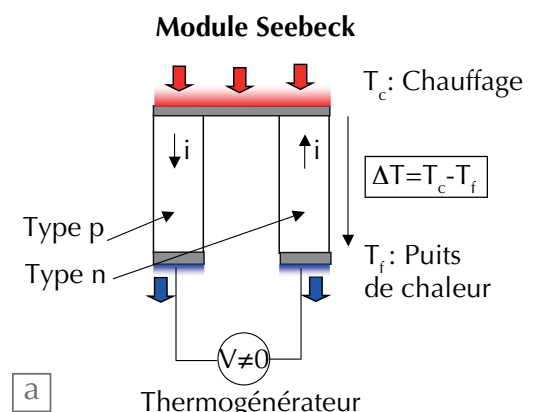

Module Peltier

Thermogénérateur

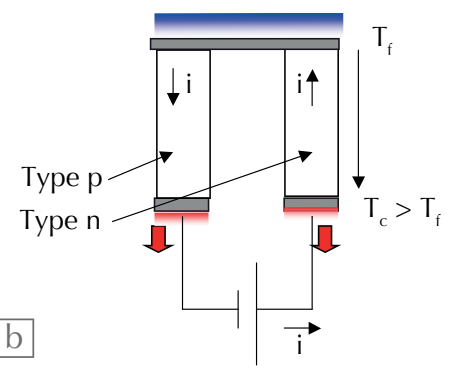

E1. (a) Module thermoélectrique en mode Seebeck. On impose une différence de température $\Delta \mathrm{T}$ aux bornes de deux matériaux dopés $p$ et $\mathrm{n}$, ce qui induit une différence de potentiel $\Delta V$ permettant de générer un courant i dans le circuit extérieur branché aux bornes du thermogénérateur.

(b) Module thermoélectrique en mode Peltier. Un courant électrique i traverse le module, permettant d'induire un refroidissement du côté de la source froide $\mathrm{T}_{\mathrm{f}}$.

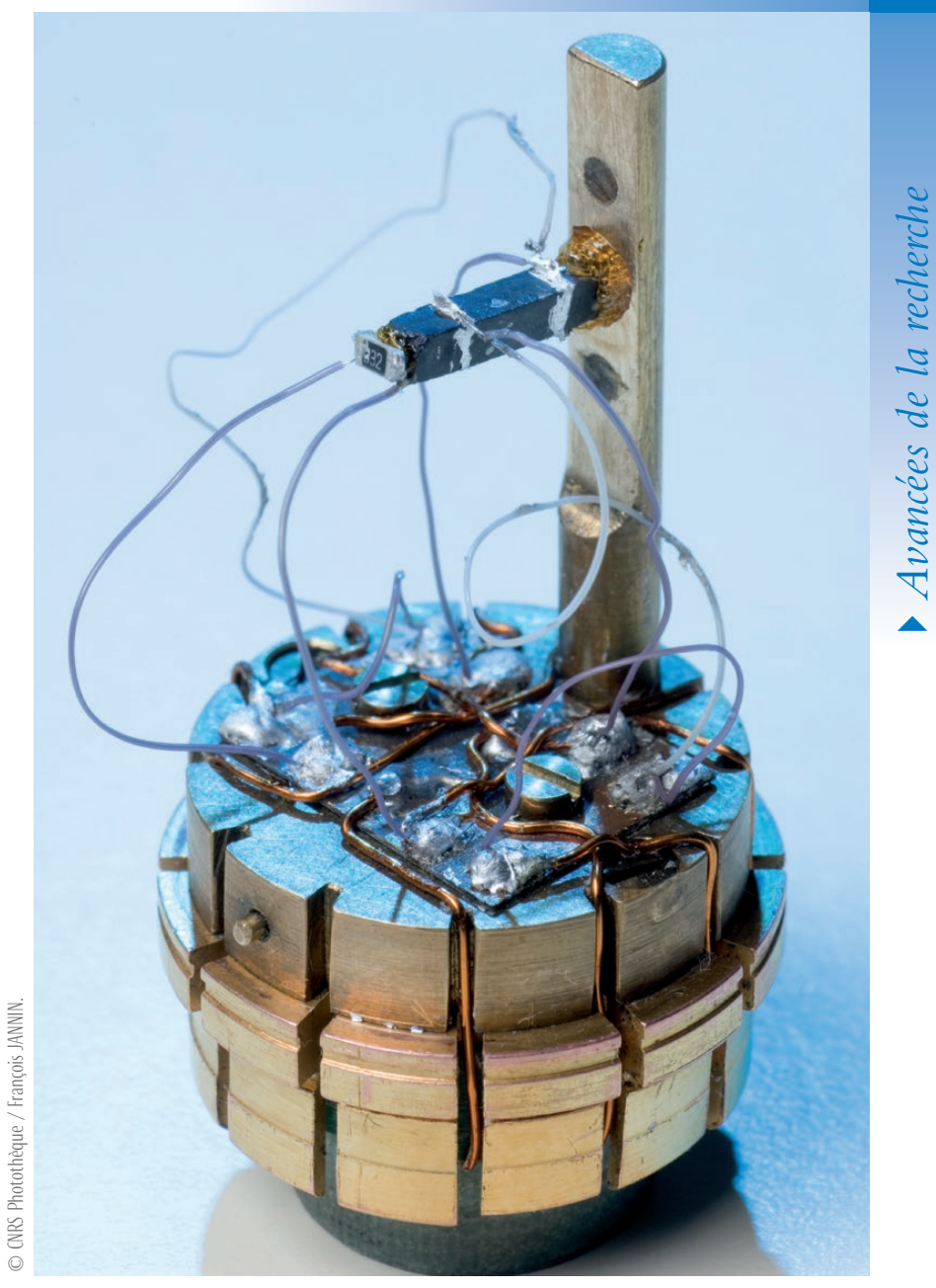

Porte-échantillon utilisé au CRISMAT pour des mesures de thermoélectricité sur une céramique.

\section{Effet Seebeck et structure électronique}

Dans un solide, les porteurs de charge (trous ou électrons) peuvent être décrits par leur densité d'états $n(E)$, qui définit le nombre de particules (par unité d'énergie) possédant une énergie $E$. $E_{F}$, l'énergie de Fermi, est l'énergie maximale des particules. Les densités $d^{\prime}$ état $\mathrm{n}(\mathrm{E})$ caractéristiques $d^{\prime} u n$ métal et d'un semi-conducteur sont schématisées sur la figure E2. On peut montrer que le coefficient Seebeck d'un métal, dans un modèle semi-classique, est donné par la formule de Mott :

$$
\mathrm{S}=\frac{\pi^{2} \mathrm{k}_{\mathrm{B}}^{2}}{3 \mathrm{e}} \mathrm{T}\left(\frac{\partial \ln \sigma(\mathrm{E})}{\partial \mathrm{E}}\right)_{E=E_{F}}
$$

avec $\mathrm{T}$ la température et $\sigma(\mathrm{E})=\mathrm{en}(\mathrm{E}) \mu(\mathrm{E})$ la conductivité électrique (e est la charge des porteurs et $\mu(E)$ leur mobilité). $S$ est donc très sensible aux porteurs dont l'énergie est proche de $\mathrm{E}_{\mathrm{F}}$. Sa valeur et son signe dépendent directement de leur mobilité, de leur nature (trous ou électrons, selon le remplissage des bandes) et de la densité d'états. Il sera maximal si $n(E)$ présente une pente élevée $d n(E) / d E$.
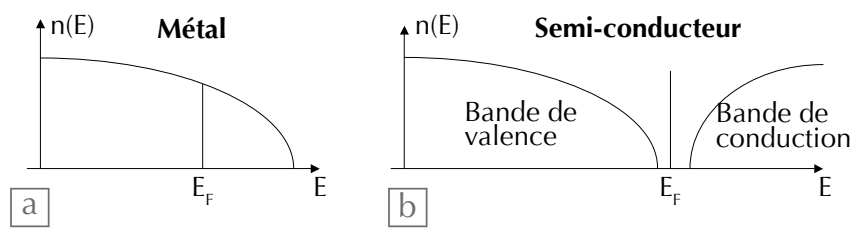

E2. Densité d'états électroniques $n(E)$ en fonction de l'énergie $E$ pour un métal (a) et pour un semi-conducteur (b). EF est l'énergie de Fermi. Dans le cas (a), le coefficient Seebeck dépend de la dérivée $(\mathrm{dn}(\mathrm{E}) / \mathrm{dE})_{\mathrm{E}=\mathrm{E} \text {. }}$ En thermoélectricité, les meilleurs matériaux présentent une énergie de Fermi proche des bords de bande de conduction ou de valence (semi-conducteurs dégénérés ou semi-métaux). 


\section{Le facteur de mérite ZT}

encadré 3

Un module thermoélectrique est constitué de nombreux couples de matériaux (tels que ceux représentés dans les figures de l'encadré 1), I'un dopé en électrons et l'autre en trous, et associés en série électriquement, et en parallèle thermiquement. Pour chaque matériau inclus dans le module, on définit un "facteur de mérite ": la quantité sans dimension $Z T=S^{2} T /(\rho \kappa)$, avec $S$ le coefficient Seebeck, $\rho$ la résistivité électrique et $\kappa$ la conductivité thermique. Le facteur ZT caractérise I'efficacité du matériau, et le critère ZT $\geq 1$ est généralement retenu pour des applications.

L'efficacité $\eta$ du module dépend des propriétés thermoélectriques des matériaux dont il est constitué, ainsi que de la différence de température $\Delta T=T_{C}-T_{f}$ auquel il est soumis, via la formule :

$$
\eta_{\max }=\frac{T_{c}-T_{f}}{T_{c}} \frac{\sqrt{1+Z T_{m}}-1}{\sqrt{1+Z T_{m}}+\left(T_{c} / T_{\mathrm{f}}\right)}
$$

où $T_{m}$ est la température moyenne et $Z T_{m}$ est proche du facteur de mérite moyen des matériaux. Cette efficacité peut se trouver fortement réduite en cas de mauvais contacts électriques ou thermiques, qui limitent l'établissement des gradients thermiques ou qui génèrent de la dissipation par effet Joule.

Les trois grandeurs $S, \rho$ et $\kappa$ sont liées via la concentration de porteurs (fig. E3), et il faut trouver un compromis entre elles pour maximiser ZT. Dans un métal, $\rho$ est très faible, mais $S$ est typiquement de quelques $\mu \mathrm{V} / \mathrm{K}$, rendant la grandeur $S^{2} / \rho$ (appelée " facteur de puissance ») très faible. Inversement, dans un semi-conducteur ou un isolant, $S$ est très élevé (quelques $\mathrm{mV} / \mathrm{K}$ ), mais la très forte valeur de $\rho$ rend aussi le rapport $S^{2} / \rho$ quasiment nul. Généralement, il existe un taux de porteurs optimal $\left(\sim 10^{20} \mathrm{~cm}^{-3}\right)$ où le terme $\mathrm{S}^{2} / \rho$ est maximal (fig. E3) : il s'agit de matériaux présentant un comportement de type "semi-conducteur dégénéré » ou « semi-métal ». La figure E4 présente les valeurs de ZT des meilleurs matériaux, dopés aux électrons ( $\mathrm{S}$ négatif) ou aux trous ( $\mathrm{S}$ positif).

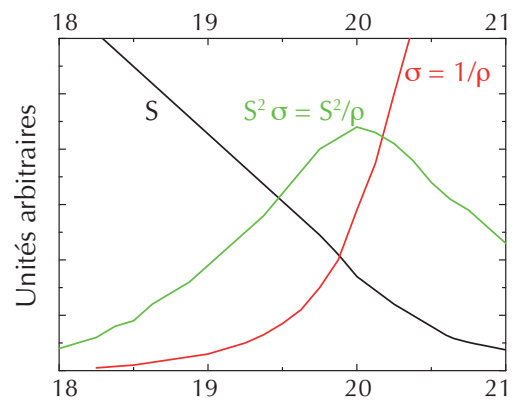

Log (concentration de porteurs par $\mathrm{cm}^{3}$ )

E3. Évolution du coefficient Seebeck $S$, de la conductivité électronique $\sigma(=1 / \rho)$ et du facteur de puissance $S^{2} / \rho$ en fonction de la concentration de porteurs.
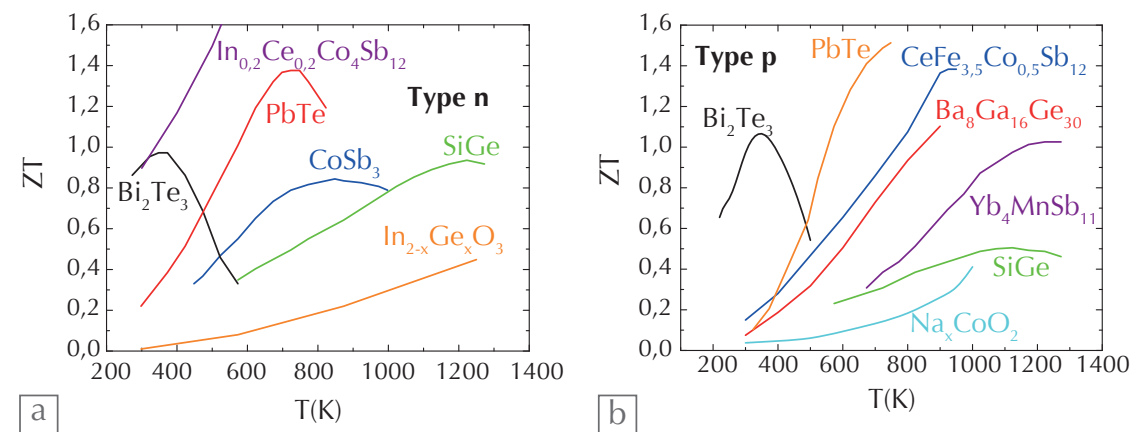

E4. Variation en fonction de la température du facteur de mérite $Z$ T pour quelques-uns des meilleurs matériaux thermoélectriques, dopés aux électrons (a) et aux trous (b). (D’après C. Godart, " Matériaux à effets thermoélectriques ", Dossier des Techniques de l'Ingénieur (juin 2009) et J. Snyder, www.thermoelectrics.caltech.edu/thermoelectrics/index.html .)
>>

sont très fortement liées entre elles via la concentration de porteurs, et varient de façon contraire (encadré 3). Il existe classiquement une plage de dopage de $\sim 10^{19}-10^{21}$ porteurs par $\mathrm{cm}^{3}$ pour optimiser ZT, et la recherche de matériaux thermoélectriques s'est longtemps focalisée sur des semi-conducteurs fortement dopés ou des semi-métaux, tels que SiGe (pour des applications spatiales avec $\mathrm{T}_{\mathrm{c}} \sim 1000^{\circ} \mathrm{C}$ ) ou $\mathrm{Bi}_{2} \mathrm{Te}_{3}$ (pour des applications à température ambiante).

Ces matériaux « classiques » de la thermoélectricité présentent des valeurs de ZT proches de 1 , mais leur utilisation peut être relativement contraignante et restreinte : problèmes d'oxydation pour $\mathrm{SiGe}$, rareté du tellure qui empêche d'envisager l'utilisation de $\mathrm{Bi}_{2} \mathrm{Te}_{3}$ pour des applications à grande échelle... Il est donc nécessaire de poursuivre la recherche de nouveaux matériaux thermoélectriques afin d'augmenter ZT, et de trouver des matériaux facilement utilisables à l'air, constitués d'éléments abondants et non toxiques. Cette recherche a connu un fort regain d'intérêt depuis le début des années 1990, suite à différentes propositions décrites dans cet article.

\section{Comment optimiser le facteur de mérite ZT ?}

Les effets thermoélectriques combinent des effets électriques et thermiques et, pour améliorer les performances thermoélectriques, il faut optimiser ces deux effets indépendamment, en minimisant la conductivité thermique $\kappa$ et en maximisant le facteur de puissance $\mathrm{S}^{2} / \rho$.

\section{1 - Réduction de la conductivité thermique}

La conductivité thermique est la somme de deux termes (conduction par les électrons/ trous et conduction par les modes de vibration des atomes, les phonons) :

$$
\kappa=\kappa_{\text {électronique }}+\kappa_{\text {phonons }} \text {. }
$$

Le terme $\kappa_{\text {électronique étant inversement }}$ proportionnel à $\rho$ (qui doit rester faible), le seul moyen de diminuer $\kappa$ est donc de diminuer $\kappa_{\text {phonons. }}$ L'approche dite $d u$ " verre de phonons / cristal d'électrons » a été proposée par Glen Slack en 1995 [2]. Il s'agissait de réduire $\kappa_{\text {phonons }}$ sans augmenter $\kappa_{\text {électronique, en introduisant dans }}$ différentes familles de "matériaux à cages " des atomes lourds. Ces derniers modifient les modes de vibration des atomes dans la structure cristallographique, réduisant la 


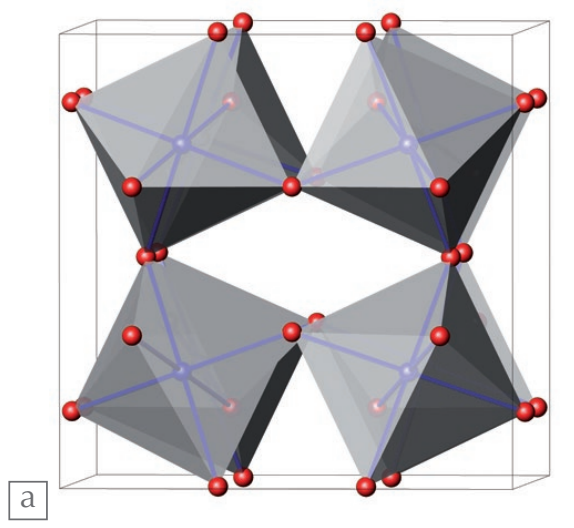

b

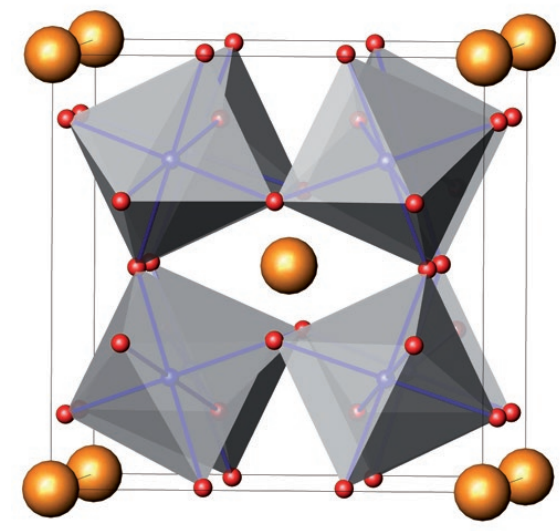

1. Structure cristallographique de (a) la skutterudite $\mathrm{CoSb}_{3}$, et (b) la skutterudite remplie $\mathrm{AC}_{4} \mathrm{Sb}_{12}$ avec, en rouge l'antimoine, en bleu l'élément de transition ( $C_{0}$, Fe), et en jaune l'atome lourd ( $A=\operatorname{Pr}$, Ce, La...).

propagation des phonons et donc $\kappa_{\text {phonons }}$. L'une des plus célèbres structures "à cages " est celle des skutterudites, matériaux de formule générique $\mathrm{MSb}_{3}$ avec $\mathrm{M}=\mathrm{Co}$ ou $\mathrm{Fe}$, dans lesquels un atome lourd $\mathrm{A}$ est introduit $\left(\mathrm{AM}_{4} \mathrm{Sb}_{12}\right)$ (fig. 1). Il a ainsi été montré, dès 1995, que l'insertion du cérium dans $\mathrm{FeSb}_{3}$ entraîne une forte réduction de $\mathrm{\kappa}$ à $300 \mathrm{~K}$, d'environ $10 \mathrm{Wm}^{-1} \mathrm{~K}^{-1}$ pour $\mathrm{FeSb}_{3}$ à $1 \mathrm{Wm}^{-1} \mathrm{~K}^{-1}$ dans $\mathrm{CeFe}_{4} \mathrm{Sb}_{12}$ [3]. $\mathrm{La}$ valeur de $\mathrm{ZT}$, initialement proche de 0,2 dans $\mathrm{CoSb}_{3}$, peut atteindre 1,2 - 1,4 à $1000 \mathrm{~K}$ dans les skutterudites " remplies " (fig. E4b). Il faut noter que même si la conductivité thermique est réduite d'un facteur 10, ZT n'est pas multiplié par 10, car l'insertion d'un atome lourd, outre son effet sur les phonons, peut aussi modifier $S$ et $\rho$, via un effet de dopage. Le choix de l'élément d'insertion doit donc prendre en compte tant l'aspect thermique que l'aspect électrique.

Le phénomène de réduction de $\kappa$ est observé dans d'autres familles prometteuses de composés à cages, comme les clathrates à base de germanium ou de silicium. Des conductivités thermiques extrêmement faibles ont été rapportées, en particulier pour $\mathrm{Ba}_{8} \mathrm{Ga}_{16} \mathrm{Sn}_{30}\left(\kappa \sim 0,5 \mathrm{Wm}^{-1} \mathrm{~K}^{-1}\right.$ à $100 \mathrm{~K})$. Ces valeurs et la dépendance en température de $\kappa(T)$ sont très proches de celles obtenues pour des verres [4]. Dans tous ces matériaux, l'effet de réduction de $\kappa$ est clairement observé, et les études en cours visent à optimiser le choix et la concentration de l'atome lourd. L'apport de la diffusion inélastique de neutrons est primordial, afin de mieux comprendre comment les modes de phonons sont affectés [5].
Dans cette recherche de "verres de phonons / cristal d'électrons ", une autre approche peut être utilisée. À partir d'un bon matériau thermoélectrique comme $\mathrm{Bi}_{2} \mathrm{Te}_{3}$, il est possible, en modifiant la microstructure (insertion d'inclusions micrométriques, par exemple), de réduire $\kappa$ sans affecter $\rho$, si le libre parcours moyen des électrons est plus faible que celui des phonons. En jouant sur les inclusions et sur des effets de joints de grain, de fortes réductions de $\kappa$ ont été obtenues, conduisant à un ZT record de 2,2 à $900 \mathrm{~K}$ dans $\mathrm{PbTe}$ [6]. À une échelle encore plus petite, la réalisation de nanostructures telles que des super-réseaux (empilements de différentes couches d'épaisseur nanométrique) ou des nanofils est également explorée. L'un des grands défis du domaine est la mesure des propriétés thermoélectriques dans ce type de nanostructure ; des techniques spécifiques ont été développées, telle que la thermoréflectance, pour sonder la conductivité thermique et les effets d'interfaces dans les super-réseaux [7].

\section{2 - Augmentation du facteur de puissance}

Historiquement, il a été proposé dès 1993 que la nanostructuration de matériaux thermoélectriques allait permettre de fortement augmenter la valeur de ZT, en jouant sur le facteur de puissance. En effet, la nanostructuration peut en théorie induire des densités d'états $\mathrm{n}(\mathrm{E})$ présentant des pics, associées à une forte augmentation de S, via la formule de Mott (encadré 2) [8]. Cette idée a été testée dans de nombreux matériaux synthétisés sous forme de superréseaux ou de nanofils. Une augmentation de $\mathrm{S}$ a effectivement été observée dans des nanofils de bismuth : S est multiplié par 1000 en passant du Bi "volumique " à des nanofils d'un diamètre de $9 \mathrm{~nm}$ [9]. Cependant, dans la plupart des cas, l'augmentation de $\mathrm{ZT}$ vient principalement de la réduction de la conductivité thermique décrite dans le paragraphe précédent, alors que l'effet sur le facteur de puissance $S^{2} / \rho$ n'est pas aussi clairement établi.

Une autre piste pour augmenter S est d'utiliser des matériaux à fortes corrélations électroniques. Parmi ceux-ci, les oxydes n'étaient traditionnellement pas étudiés en thermoélectricité, leur résistivité étant trop élevée. En 1997, il a cependant été montré qu'un coefficient Seebeck élevé $(\sim 100 \mu \mathrm{V} / \mathrm{K})$ était obtenu dans un oxyde relativement métallique, $\mathrm{Na}_{\mathrm{x}} \mathrm{CoO}_{2}(\mathrm{x} \sim 0,7)$ [10], alors que le facteur $\mathrm{S}^{2} / \rho$ à $300 \mathrm{~K}$ était équivalent à celui de $\mathrm{Bi}_{2} \mathrm{Te}_{3}$. Comme, de plus, $\kappa$ est faible, cet oxyde a été classé dans la catégorie "verre de phonons / cristal d'électrons". Ce résultat a entraîné une forte activité dans la recherche de nouveaux oxydes thermoélectriques : en effet, ils peuvent être utilisés à haute température, sous air pour la plupart d'entre eux, et peuvent être constitués d'éléments abondants et non toxiques. Dans ces matériaux, la bande de conduction est relativement étroite, et les modèles utilisés pour les matériaux thermoélectriques classiques ne s'appliquent pas. Une forte augmentation de la pente $\mathrm{dS} / \mathrm{dT}$, résultant des corrélations électroniques, peut être observée à basse température [11]. De plus, pour ces oxydes, il est possible d'évaluer la limite du coefficient Seebeck S à haute température via la formule de Heikes (encadré 4), dans laquelle S est une mesure de l'entropie associée à chaque porteur. Le métal de transition (fer, cobalt, manganèse...) est source d'entropie via son spin et son remplissage des orbitales, entropie qui contribue à augmenter $\mathrm{S}$ [12]. La résistivité des oxydes restant souvent trop élevée, des sulfures, séléniures ou oxyséléniures, plus covalents, sont actuellement étudiés afin de réduire $\rho$, en essayant de conserver des corrélations relativement fortes.

Il faut noter que dans ces oxydes, le taux de porteurs est plus élevé que pour les matériaux thermoélectriques classiques $\left(\sim 10^{22} \mathrm{~cm}^{-3}\right)$, ce qui montre que leurs propriétés sont souvent très différentes de celles décrites par la formule de Mott, et que ces oxydes nécessitent des modèles spécifiques, toujours en cours de développement [13]. 


\section{Conclusion}

La thermoélectricité connaît actuellement un fort regain d'intérêt comme éventuelle source d' "énergie propre ", les sources de chaleur perdue étant abondantes; mais les applications à grande échelle ne seront réalisables que via l'optimisation des matériaux et des modules. Cette recherche de nouveaux matériaux nécessite un travail très interdisciplinaire, alliant chimistes, physiciens, thermiciens... et a déjà permis une forte augmentation des performances, puisque des valeurs de ZT proches de 1,9 à 2,2 ont été rapportées. Ces exemples montrent qu'il est intéressant d'aller au-delà de la physique classique des semi-conducteurs, et qu'une compréhension plus microscopique des phénomènes est à présent envisageable grâce aux progrès des techniques expérimentales (techniques de synthèse pour modifier la micro- ou la nanostructure, étude de la structure de bandes par photoémission, mesure du spectre de phonons par diffusion de neutrons...) et aux développements théoriques récents (développement de la formule de Kelvin, amélioration des calculs de structure de bandes...).

\section{Références}

1- Thermoelectrics Handbook : macro to nano, édité par D.M. Rowe, CRC Press, Taylor and Francis (2006) ; B. Lenoir et al., «Thermoélectricité : des principes aux applications ", dossier des Techniques de l'Ingénieur (mai 2010) ; C. Goupil et al., « Thermoélectricité Thermodynamique et applications ", dossier des Techniques de I'Ingénieur (avril 2013).

2• Glen A. Slack, CRC Handbook of Thermoelectrics, chapitre 34, édité par D.M. Rowe, CRC Press (1995).

3• D. Morelli et G. P. Meisner, J. Appl. Phys. 77 (1995) 3777.

4• M.A. Avila et al., Appl. Phys. Lett. 92 (2008) 041901.

5• M.M. Koza et al., Phys. Rev. B 84 (2011) 014306.

6• K. Biswas et al., Nature 489 (2012) 414.

7• M. M. Rojo et al., J. Appl. Phys. 113 (2013) 054308.

8• L.D. Hicks et M.S. Dresselhaus, Phys. Rev. B 47 (1993) 12727.

9• J.P. Heremans et al., Phys. Rev. Lett. 88 (2002) 216801. 10• I. Terasaki et al., Phys. Rev. B 56 (1997) R12685.

11 K. Behnia, D. Jaccard et J. Flouquet, J. Phys.: Condens. Matter 16 (2004) 5187.

12• S. Hébert et al., Phys. Status Solidi A 210 (2013) 67.

13• L-F. Arsenault et al., Phys. Rev. Lett. 110 (2013) 086401.

\section{Pouvoir thermoélectrique des oxydes de métaux} de transition : la formule de Heikes encadré 4

Pour des matériaux possédant des bandes de conduction très étroites (tels ceux à fortes corrélations électroniques), les approches semi-classiques ne sont plus valables et des modèles spécifiques ont été développés. On peut montrer qu'à haute température, le coefficient Seebeck $\mathrm{S}$ est directement une mesure de l'entropie par charge dans le système ; pour maximiser $\mathrm{S}$, il faut alors maximiser cette entropie. L'intérêt des oxydes est que les métaux de transition tels que le cobalt, le fer, le manganèse... peuvent générer de l'entropie via leurs spins et leur dégénérescence orbitalaire. Le coefficient Seebeck peut être bien décrit à haute température par la formule de Heikes, qui dépend des dégénérescences de spin et d'orbitale des ions de métaux de transition. Un exemple est montré dans la figure E5 : dans le cas d'un oxyde à valence mixte $\mathrm{Co}^{3+} / \mathrm{Co}^{4+}$, au terme lié au taux de porteurs (courbe noire, $\beta=1$ ) s'ajoute le terme de dégénérescence de spin et d'orbitale (courbes rouge ou verte). La validité de la formule de Heikes est théoriquement restreinte aux hautes températures, mais un développement plus rigoureux (formule de Kelvin) montre qu'elle représente une bonne approximation de $\mathrm{S}$ dans une large gamme de températures.

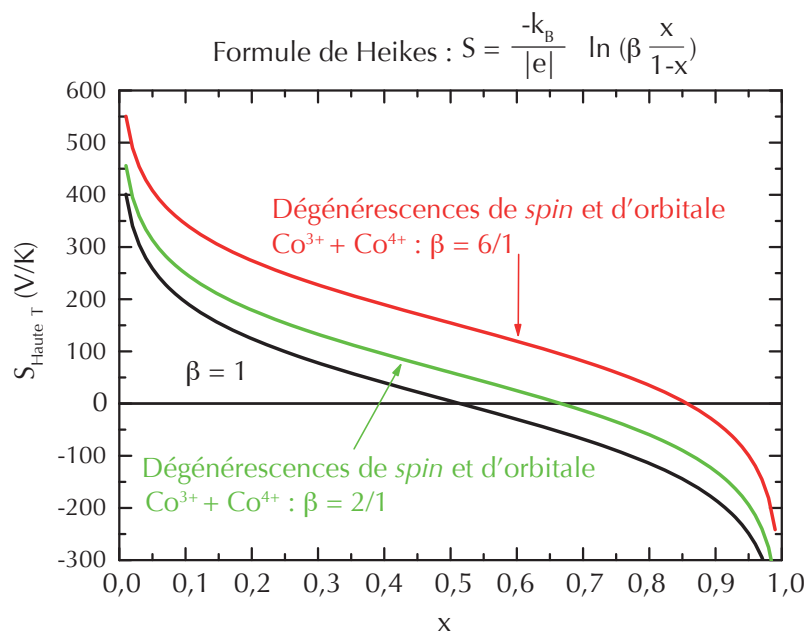

Ion $\mathrm{Co}^{4+}$

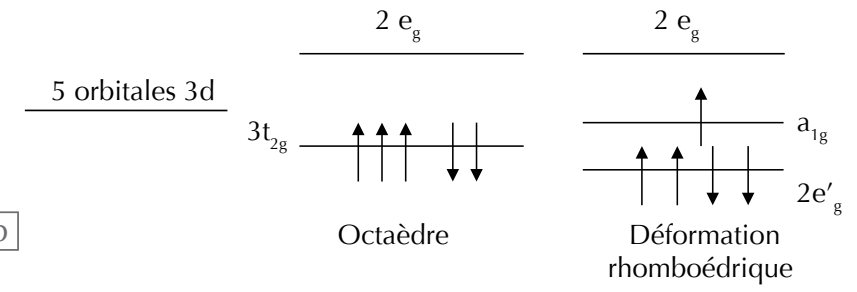

E5. (a) Coefficient Seebeck $S$ à haute température T, en fonction du taux x d'ions $\mathrm{C}^{4+}$, calculé par la formule de Heikes pour des oxydes de cobalt présentant une valence mixte $\mathrm{Co}^{3+} / \mathrm{Co}^{4+}$, et (b) les dégénérescences de spin et d'orbitales associées.

Pour un ion cobalt en environnement octaédrique, les cinq orbitales $3 d$ sont séparées en deux orbitales $e_{q}$ et trois orbitales $t_{2 g}$. Une déformation des octaèdres lève la dégénérescence des orbitales $t_{2 g}$ en deux niveaux $\mathrm{e}_{\mathrm{g}}^{\prime}$ et un niveau $\mathrm{a}_{1 \mathrm{~g}}$. Pour l'ion $\mathrm{C}^{3+}\left(3 \mathrm{~d}^{6}\right)$, il n'y a pas de dégénérescence, les trois orbitales $\mathrm{t}_{2 \mathrm{~g}}$ sont occupées. Pour l'ion $\mathrm{C}_{0}{ }^{4+}\left(3 d^{5}\right)$, la dégénérescence est de 6 si les trois orbitales $t_{2 g}$ sont dégénérées (un trou sur trois orbitales, avec spin $\uparrow$ ou spin $\downarrow$ ), et de 2 s'il y a levée de dégénérescence (un trou sur l'orbitale $\mathrm{a}_{1 \mathrm{~g}}$, avec spin $\uparrow$ ou spin $\downarrow)$. Donc, $\beta=$ dégénérescence $\left(C 0^{4+}\right)$ /dégénérescence $\left(C_{0}^{3+}\right)=6 / 1$ (sans déformation) ou 2/1 (avec déformation). S haute T $(\mathrm{x})$ est alors donné par les courbes rouge et verte de la figure (a).

- Pour en savoir plus

Cours d'Antoine Georges au Collège de France, printemps 2013 www.college-de-france.fr/site/antoine-georges/course-2012-2013.htm 